\title{
Survey of terahertz metamaterial devices
}

\author{
Withawat Withayachumnankul ${ }^{1,2}$ and Derek Abbott ${ }^{1}$ \\ ${ }^{1}$ School of Electrical \& Electronic Engineering, \\ The University of Adelaide, Adelaide, SA 5005, Australia \\ ${ }^{2}$ Department of Information Engineering, Faculty of Engineering, \\ King Mongkut's Institute of Technology Ladkrabang, Bangkok 10520, Thailand
}

\begin{abstract}
Metamaterials have arisen in an attempt to engineer the electromagnetic properties of natural substances. It has been acknowledged that the emergence of metamaterials has implications to nearly all branches of science and engineering exploiting the electromagnetic radiation. This paper reviews seminal work of metamaterials from the vision to the realisation of subwavelength elements that contribute to varieties of electric and magnetic responses. Emphasis is given to the significance and opportunity of this new class of material augmenting terahertz technology. Although now there remain major milestones that scientists and engineers need to conquer, the future of this cutting-edge material technology is very bright.
\end{abstract}

Keywords: terahertz; T-rays; metamaterial; split-ring resonator

\section{INTRODUCTION}

A metamaterial is a composite basically composed of conductive elements, of subwavelength dimension, periodically embedded in a dielectric substrate. The electromagnetic properties of metamaterials are derived mainly from the morphology of these elements rather than from bulk properties as do conventional materials. ${ }^{1}$ Since these elements are small relative to the operating wavelength, the entire metamaterial structure appears homogeneous to the radiation, and hence can be characterised by two fundamental electromagnetic measures - the permittivity and permeability.

Metamaterials can be engineered to have a wide range of electromagnetic responses at desired frequencies through the design of embedded metallic elements. This includes those responses not found in naturally occurring materials, and hence the name 'meta', which implies 'beyond' materials. In terms of the real permittivity and permeability, normal dielectrics typically occupy the domain where both the quantities are positive, as shown in Figure 1. Negative values of the permittivity are attainable in nature through radiation-plasma interaction, but the occurrence of this interaction is typically well beyond the infrared for those solid-state plasmas. Metamaterials, or more specifically, thin-wire lattices, can dilute the plasma cloud and depress the plasma frequency to a lower part of the spectrum. ${ }^{2}$ Negative values of the permeability are rare and obtainable from magnetic resonances in ferromagnets only at sub-microwave frequencies. Metamaterials, such as split-ring resonators ${ }^{3}$ and parallel strips, exhibit magnetic dipoles in response to magnetic fields, and push the magnetic resonance towards the optical regime. ${ }^{4}$

Negative refractive index is a particular electromagnetic feature offered by metamaterials. The origin of negative index metamaterials (NIMs) dates back to 1968 when Veselago ${ }^{5}$ conceived the existence of a material with its permittivity and permeability being together negative (see Figure 1). Although such a material was not discovered at that time, Veselago, in his revolutionary paper, predicted some spectacular effects of NIMs, including the reverse Doppler shift, reverse Chěrenkov radiation, negative refraction, etc. A double-negative material has never been found in nature, because the negative bands of the permittivity and permeability are relatively narrow and stand well apart in the spectrum. ${ }^{6}$ It was not until 2000 that a NIM was realised for the first time from metamaterials at microwave frequencies by Smith et al. ${ }^{7}$ Since then a variety of applications exploiting NIMs have been proposed at virtually every band of the electromagnetic spectrum.

Corresponding author: W. Withayachumnankul (withawat@eleceng.adelaide.edu.au)

Smart Structures, Devices, and Systems IV, edited by Said Fares Al-Sarawi,

Vijay K. Varadan, Neil Weste, Kourosh Kalantar-Zadeh, Proc. of SPIE Vol. 7268, $72681 Z$

(c) 2008 SPIE $\cdot$ CCC code: $0277-786$ X/08/\$18 $\cdot$ doi: $10.1117 / 12.823538$ 


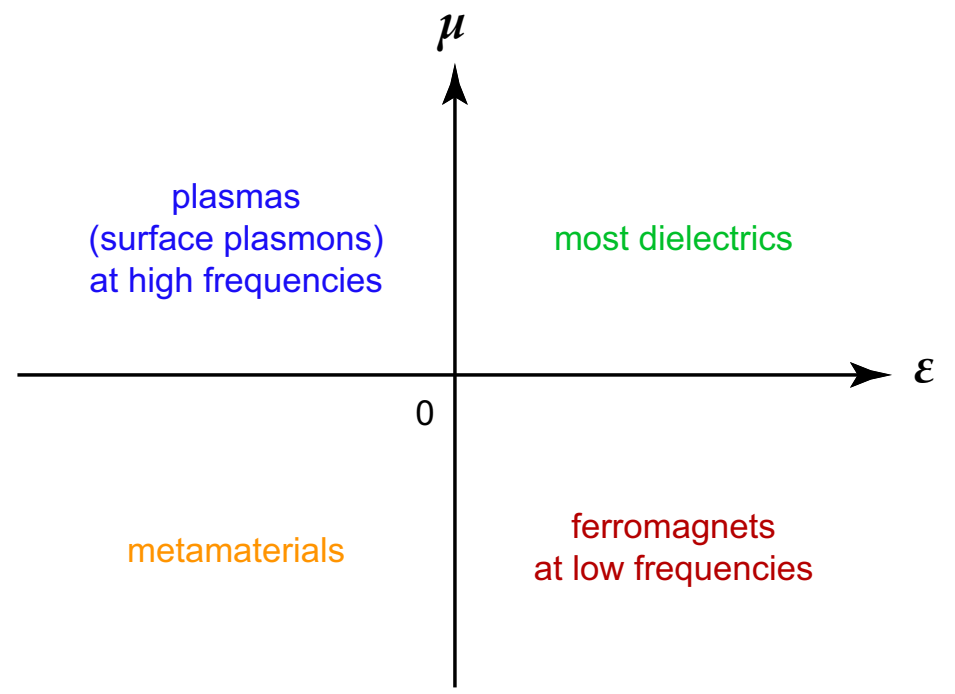

Figure 1. Diagram of real permittivity and permeability. Each quadrant is occupied by a specific class of naturally existing materials, except for the third quadrant where both the permittivity and permeability are negative at the same frequency. This quadrant is realised by negative-index metamaterials.

Even though metamaterials provide customisable electromagnetic responses, they should not be confused with photonic crystals although the two classes of structures share some similar behaviour and consist of periodic units. In terms of the feature dimension, metamaterials embrace subwavelength elements, in contrast to photonic crystals that have feature sizes rational and comparable to the operating wavelength. In addition, coupling between electrons in conductive subwavelength elements and the electromagnetic radiation determines the response of metamaterials, whilst reflection and interference of the electromagnetic radiation by periodic structures determine the response of photonic crystals.

Opening a new regime of the electromagnetic response, metamaterials offer immense opportunities in improving existing optical designs along with exploring unprecedented applications. Relevant research breakthroughs and visions proposed so far encompass: superlenses breaking the diffraction-limited image resolution, ${ }^{8}$ biosensors sensitive to small changes in the amount and response of a sample, ${ }^{9}$ magnetic wires retrofittable into existing MRI machines for better magnetic coupling, ${ }^{10}$ invisibility cloaks for hiding an object from detection, ${ }^{11-13}$ and many more exciting ideas to come. Because metamaterial research has started just recently, fundamental studies, novel designs, and advance applications of metamaterials have yet to be explored. It is expected that this research field will have great impact on both the physics and engineering disciplines.

In this paper a concise review on metamaterials is presented, with particular interest in the significance and opportunities of materials operating in the terahertz spectrum. Background in electric and magnetic metamaterials is discussed in Section 2 and 3, respectively. NIMs, obtainable from the combination of electric and magnetic metamaterials, are elucidated in Section 4. The significance of terahertz metamaterials is thoroughly discussed in Section 5. A variety of terahertz metamaterial devices proposed so far are surveyed in Section 6.

\section{ELECTRIC METAMATERIALS}

The electric response of natural conductive materials typically takes place at high frequencies, i.e. at visible or UV for metals. This is evident from the electric plasma frequency, $\omega_{\mathrm{ep}}$, which can be formulated as

$$
\omega_{\mathrm{ep}}^{2}=\frac{n e^{2}}{\epsilon_{0} m_{\mathrm{eff}}},
$$

where $n$ is the electron density, $e$ is the electron charge, $\epsilon_{0}$ is the vacuum permittivity, and $m_{\text {eff }}$ is the electron effective mass. For example, gold that has an electron density of $5.9 \times 10^{22} \mathrm{~cm}^{-3}$ will have a plasma frequency falling at approx. $138 \mathrm{~nm}$ or within the far UV. 


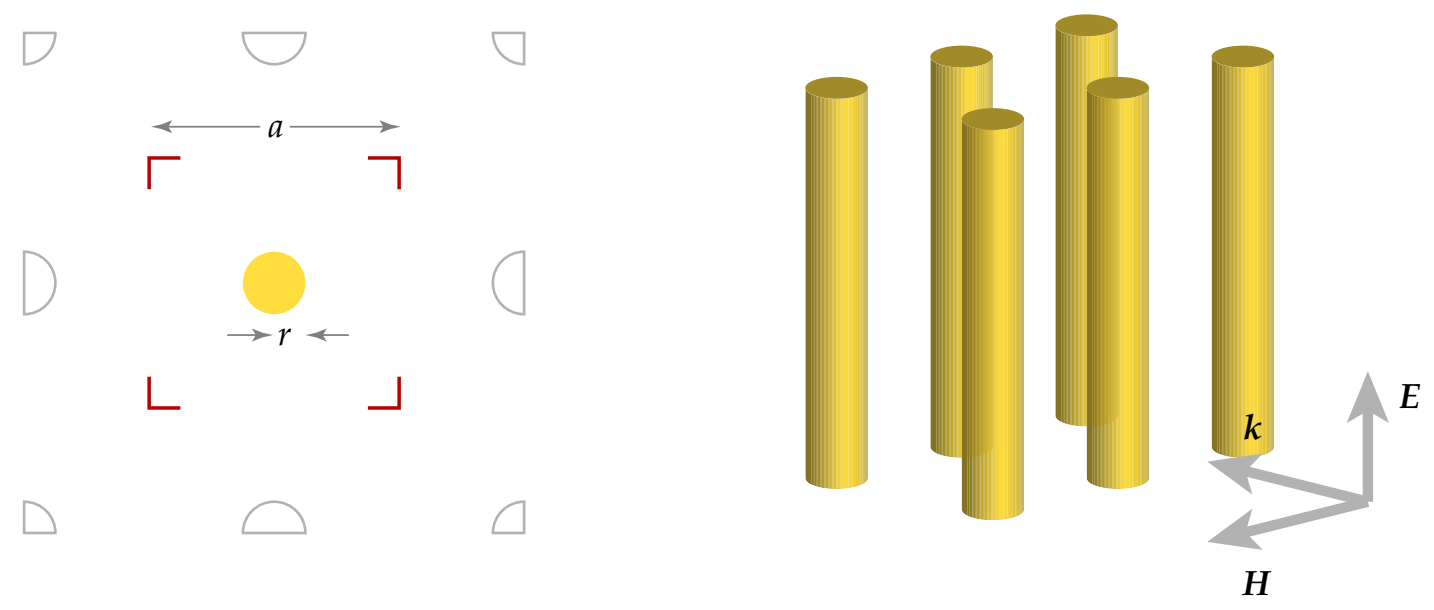

(a) metamaterial footprint

(b) 3D impression

Figure 2. Lattice of thin metallic wires. With the electric field parallel to the wires, the structure exhibits a Drude electric response with a plasma frequency governed by its geometry. The red markers in the footprint delimit a unit cell.

Apparently, to achieve the electric response at a lower frequency range, e.g. in the microwave region, the plasma frequency must be modified. According to Equation 1 the plasma frequency can be reduced through a change in the electron density and effective mass. Pendry et al. ${ }^{2}$ theoretically proposed that a metamaterial made of a lattice of thin metallic wire, similar to that shown in Figure 2, can bring down plasma frequency to the gigahertz range. In such a structure, the electron density $n$ is diluted due to the sparseness of metal in a unit cell. Furthermore, the electron effective mass is intensified because of the apparent mutual inductance of the wires that exerts the force on electrons. From the analysis, given that $a$ is the lattice spacing and $r$ is the wire radius, the plasma frequency of the structure now becomes ${ }^{2,14}$

$$
\omega_{\mathrm{ep}}^{2}=\frac{2 \pi c_{0}^{2}}{a^{2} \ln (a / r)}
$$

where $c_{0}$ is the velocity of light in vacuum. It is clear that the plasma frequency of the structure can be manipulated through the dimensions, $a$ and $r$. At its operational frequency where the wavelength is much larger than a unit length, the structure appears homogeneous. Thus the structure can be characterised by an effective permittivity that takes on a Drude model,

$$
\epsilon_{\mathrm{eff}}=1-\frac{\omega_{\mathrm{ep}}^{2}}{\omega^{2}+j \Gamma \omega}
$$

where $\Gamma$ is responsible for the propagation loss. At $\omega<\omega_{\text {ep }}$ the permittivity becomes negative.

The analysis proposed by Pendry et al. ${ }^{2}$ was later confirmed by numerical simulations and experiments in the microwave regime. ${ }^{14}$ In the experiments, a 2D lattice of wires aligned at right angle was constructed from goldcoated tungsten wires embedded in polystyrene sheets with the feature sizes $a$ and $r$ of the order of millimetres and micrometres, respectively. The measurement showed that the transmission is prohibited below the plasma frequency - implying the negative permittivity, and that the sparser wire distribution leads to a lower plasma frequency.

\section{MAGNETIC METAMATERIALS}

Several designs of magnetic metamaterials, including split-ring resonators (SRRs), made of nonmagnetic conductive elements were introduced by Pendry et al. ${ }^{3}$ The SRR structure is basically composed of concentric metallic rings with dielectric gaps, as illustrated in Figure 3. When the SRR is coupled to a magnetic field component 


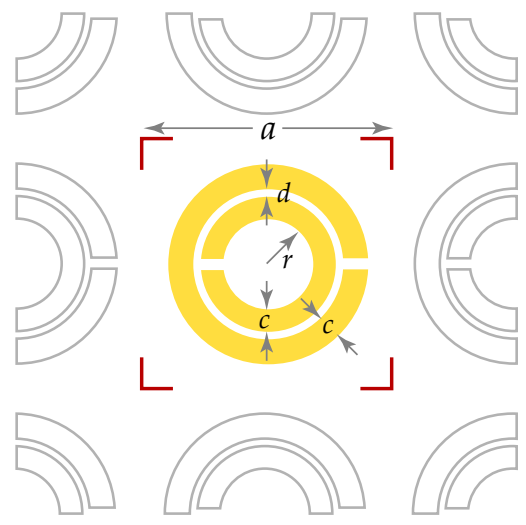

(a) metamaterial footprint

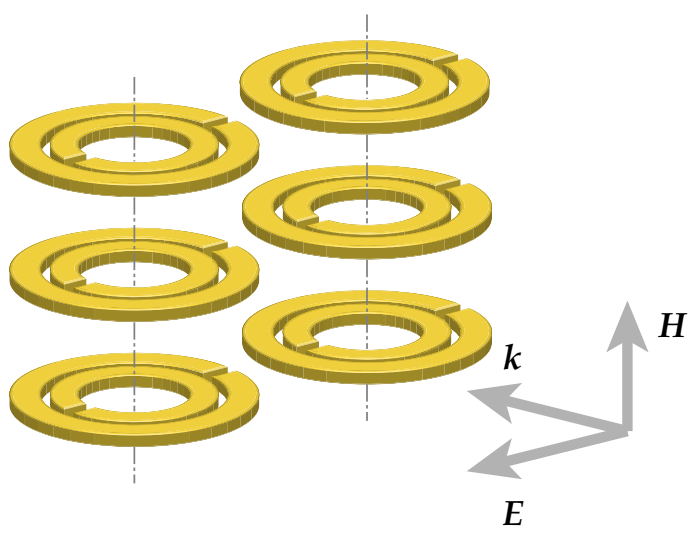

(b) 3D impression

Figure 3. Split-ring resonators. This metamaterial exhibits a Lorentzian resonance in the effective magnetic permeability, when excited by a magnetic field parallel to the stacking axis. The red markers in the footprint delimit a unit cell.

that is oscillating in the axial direction, the ring establishes current flow, which further builds the magnetic dipole parallel or antiparallel to the magnetic field. The resonance frequency of the structure is determined from the inductance of the loops and the capacitance at the gaps, which can be modelled by an equivalent LC resonator circuit. In the original model, the second smaller concentric ring increases the apparent capacitance of the SRR and thus reduces the resonance frequency. At frequencies lower than the resonance the SRR has a positive response to the magnetic field, and slightly above the resonance the response becomes negative. A metamaterial structure containing those SRRs can be described by the effective magnetic permeability with a Lorentzian model:

$$
\mu_{\mathrm{eff}}=1-\frac{\omega_{m p}^{2}-\omega_{m 0}^{2}}{\omega^{2}-\omega_{m o}^{2}+j \Gamma \omega},
$$

where $\omega_{m 0}$ and $\omega_{m p}$ are the magnetic resonance and plasma frequencies, respectively, and $\Gamma$ represents the loss in the structure. Through a proper design of SRRs, the effective magnetic permeability of the structure can become diamagnetic $\left(\mu_{\mathrm{eff}}<1\right)$, paramagnetic $\left(\mu_{\mathrm{eff}}>1\right)$, or even negative at desired frequencies.

\section{NEGATIVE REFRACTIVE INDEX METAMATERIALS}

In 1968 Veselago $^{5}$ raised a series of questions about a hypothetical material with both negative electric and magnetic responses - whether it is possible, how it interacts with the radiation, and where it can be found. Purely from the theoretical viewpoint he asserted that such a material can exist and does not violate any law of physics, and moreover it interacts with the electromagnetic wave in novel ways. Essentially, in a material with a permittivity and permeability being subzero, $\boldsymbol{E}$ and $\boldsymbol{H}$ form a left-handed vector set with respect to $\boldsymbol{k}$, i.e. the wave vector becomes negative. In contrast, the Poynting vector $\boldsymbol{S}$, indicating the propagation direction of wave energy, still forms a right-handed triplet with $\boldsymbol{E}$ and $\boldsymbol{H}$. Hence, the wavefront propagates backward in the medium, and is antiparallel with the direction of energy flow. Postulated by Veselago, this kind of medium, if realised, would exhibit a number of intriguing phenomena, including the reversed Doppler, reversed Chěrenkov radiation, and negative refraction. Furthermore, he suggested that negative permittivity and permeability are most likely occur in natural anisotropic substances, but none has been found to date. Note that the term 'lefthanded materials', inferred from the left-handed vector triplet, was adopted in early work, but this term is also used in the context of chirality and thus can be misleading. Consequently, the terms negative-index materials or double-negative materials are currently preferred.

The idea of NIMs had remained theoretical for more than 30 years since its first introduction. The advent of metamaterials with a tailored electric response ${ }^{2}$ in 1996 and a tailored magnetic response ${ }^{3}$ in 1999 pointed 


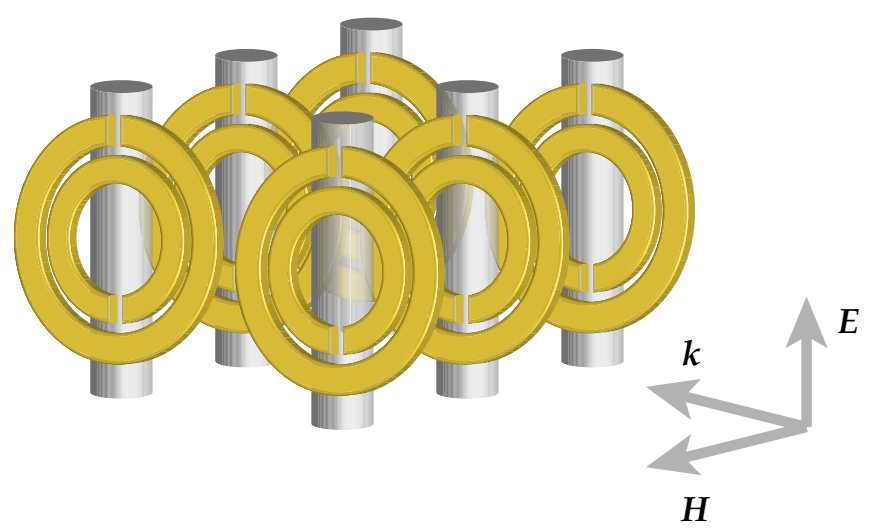

Figure 4. Negative-index metamaterial. The structure is composed of wires and SRRs, which are responsible for the electric and magnetic responses, respectively. At a narrow frequency band the permittivity and permeability can be negative, resulting in demonstration of negative refractive index.

the way towards a possible implementation of NIMs that have both negative electric and magnetic responses. The first artificial NIM was realised by Smith et al. ${ }^{7}$ in 2000, who combined SRRs and a thin-wire lattice to produce negative $\mu$ and $\epsilon$ at the microwave frequency band. At a frequency with either negative permittivity or permeability alone, the material is opaque to the radiation, but overlapping of the two leads to the presence of a passband. In the experiment of Smith et al.,$^{7}$ a periodic array of copper SRRs interleaved with thin wires is oriented in the electromagnetic field such that the magnetic field is parallel to the axis of SRRs and the electric field is parallel to the axis of wires - similar to the structure shown in Figure 4. A transmission measurement reveals a passband around $5 \mathrm{GHz}$, implying the presence of negative refractive index. The result was reproduced with similar experiments ${ }^{15,16}$ and with a refined numerical simulation. ${ }^{17}$

The structure proposed by Smith et al. ${ }^{7}$ is anisotropic, i.e. the negative refractive index appears in only one propagation direction. In order to realise extended physical consequences of NIMs, the material isotropy is vital. Based on the SRR/wire combination, a 2D-isotropic NIM is assembled from interlocked fibreglass boards with SRRs imprinted on one side and wire strips on the other side. ${ }^{18}$ Confirmed by an experiment and a transfer-matrix calculation, the 2D-isotropic passband appears around the microwave X-band as a result of negative refractive index. A similar 2D NIM cut into a wedge shape was used for direct observation of negative refraction, ${ }^{19}$ i.e. the transmitted ray refracted on the same side with the incident ray. At $10.5 \mathrm{GHz}$, the refractive index was measured to be $-2.7 \pm 0.1$.

\section{SIGNIFICANCE OF METAMATERIALS TO TERAHERTZ}

The current trend of metamaterial research aims for design of nanostructures that are capable of manipulating electromagnetic waves at the visible frequency regime. ${ }^{4,20,21}$ Although in theory metamaterial structures are physically scalable to fit a working spectral regime, approaching such a short-wavelength operation is in essence challenging in that the design needs to assist towards the fabrication process yet preserve the integrity. ${ }^{22}$ This latest effort in metamaterial research will lead to optical nanostructures that are of primary importance to communication, microscopy, and defence sectors. But looking back at lower frequency regimes, in particular around the terahertz gap, there are a number of opportunities for improvement and implementation of metamaterials that remain.

The terahertz spectrum, loosely defined between 0.1 and $10 \mathrm{THz},{ }^{23}$ is of great importance, as the frequency underlies a wide range of significant microscopic physical phenomena. In general, bulk dielectric relaxations and intermolecular motions occur in this frequency range. ${ }^{24}$ Intermolecular hydrogen bonds in crystalline solids resonate at terahertz frequencies, causing distinctive absorption features. ${ }^{25}$ Critical frequencies for Debye relaxation processes in liquids fall into the terahertz regime. ${ }^{24}$ Pure rotational transitions can be observed when polar gases are stimulated by terahertz radiation. ${ }^{26}$ Moderately doped semiconductors have their plasma frequencies and damping rates defined between 0.1 and $2 \mathrm{THz} .{ }^{27}$ The terahertz radiation is also used to 
stimulate Rabi oscillations in two-level impurity states in semiconductors, which enables the manipulation of physical qubits. ${ }^{28-30}$

At a macroscopic scale, many distinctive properties are associated with terahertz. The radition is nonionising $^{31}$ and hence favourable to applications seeking human exposure, e.g. medical diagnoses or security screening. A vast number of materials pronounce unique absorption patterns in response to terahertz, in accordance with their molecular rotational/vibrational modes. ${ }^{32}$ These absorption spectra lend themselves to substance identification. ${ }^{25,33,34}$ Non-polar, dry, and non-metallic materials, such as fabrics, woods, cardboards, and plastics, are transparent to the radiation. Hence, weapons concealed beneath clothing or products contained in packages, for example, can be seen through terahertz sensors. ${ }^{35-37}$

We have seen that the individual areas of metamaterial and terahertz research are very attractive, yet the combination of the two - metamaterials operating in the terahertz regime - has not seen much activity so far either in terms of fundamental research or advanced applications. At this point, one might raise a question about the scarceness of research on terahertz metamaterials. A response to this question may simply be related to the fact that both metamaterial and terahertz research are rather in their infancy - an efficient approach to terahertz spectroscopy ${ }^{38}$ emerged in 1989, whilst the first experiment on metamaterials ${ }^{7}$ appeared in 2000. Optical, infrared, or microwave spectroscopy systems by far outnumber novel terahertz systems, and a majority of the growing metamaterial research groups have preferably relied on the more mature spectroscopic systems.

The terahertz spectrum represents an interesting arena for metamaterial research in light of the fact that naturally occurring materials do not exhibit strong magnetic or electric responses between 1 and $3 \mathrm{THz} .{ }^{39}$ Those conventional magnetic materials tend to have resonances at frequencies below the gigahertz range, whilst metals possess resonances at frequencies beyond the mid-infrared owing to phonon modes. Thus, in this sense, the terahertz gap not only defines a region where the electromagnetic spectrum is relatively unexplored, but also a region where the electromagnetic responses of most conventional materials nearly diminish. Because of their customisable responses, metamaterials can fulfil the missing link, and become a foundation for further terahertz research. Ultimately, these metamaterials may develop into terahertz-manipulating devices that outperform their predecessors, or moreover, that are inconceivable from traditional materials.

\section{REALISATION OF TERAHERTZ METAMATERIAL DEVICES}

Scalability of metamaterials in theory suggests direct adoption of structures originally designed for operation at a specific frequency to operate at any other point across the electromagnetic spectrum. But in reality, there exist many implications, and scaling the structure alone would not yield good metamaterial performance. Although not directly related to metamaterials' responses, constituent materials, i.e. metals and dielectrics, play a major role in dissipation of the radiation energy. At the operating frequency range, here terahertz, metals need to be highly conductive and dielectrics need to be transparent to reduce the associated loss. Moreover, the fabrication process is not scalable, and low-cost is desirable. Current technologies for IC fabrication are very suitable for producing planar terahertz metamaterials, but achieving fully 3D-isotropic structures is rather complicated at the terahertz-wavelength scale. Despite that, a relatively small number of fundamental studies, involving fabrication and characterisation, have been carried out with terahertz metamaterials, ranging from magnetic metamaterials, ${ }^{40-42}$ to electric metamaterials, ${ }^{43-46}$ to NIMs. ${ }^{47-50}$ The reasonable performance of teraheretz metamaterials leads to recent realisation of communication and sensing devices, which will be discussed in details hereafter.

The existence of either negative permittivity or permeability alone in a metamaterial can be utilised for a bandstop filter, because at this spectral region no propagation mode is permitted. As discussed earlier, a lattice of metallic wires produces the negative permittivity below the plasma frequency. Hence the lattice that is designed to have a plasma frequency at the terahertz range will function as a terahertz high-pass filter. ${ }^{51}$ A structure constructed from gold-coated polymer columns confirms the appearance of a stopband below the plasma frequency when the electric field aligns in parallel to the columns. ${ }^{51}$ It is noted that the gold coating thickness is larger than the skin depth of gold, so the effect of the polymer can be ignored. Impedance matching of metamaterials can be exploited for a narrowband terahertz absorber. ${ }^{52}$ A structure comprising electric and magnetic resonators is designed such that the resonance response results in impedance matching of the structure 
to free space in a narrow frequency band. At this frequency the reflection is nearly suppressed. Inherent absorption in the structure, in addition, lessens the transmission, and hence very low terahertz energy can escape at the other side. This metamaterial absorber is suitable for constructing thermal detectors.

As the performance of existing terahertz switches/modulators proves to be impractical, metamaterials offer an alternative in dynamical manipulation of the terahertz. This concept was first exploited for a narrowband terahertz switch. ${ }^{53}$ In that work, copper SRRs are fabricated on a high-resistivity GaAs substrate, and the terahertz polarisation is aligned such that the electric resonance mode of SRRs is activated. Under the influence of an optical excitation, photo-induced free carriers are generated in the substrate, shorting the SRR gap and thus eliminating the resonance response. This implies the on state of the switch at the resonance frequency. In the absence of the optical excitation, the resonance is restored, leading to the off state. A faster terahertz switch replaces the substrate with ErAs/GaAs supperlattices with faster carrier recombination, resulting in a switching recovery time of picoseconds or shorter. ${ }^{54}$ For a terahertz switch controlled via an optical excitation, since optically-induced carriers are not localised, they interact with and absorb the terahertz radiation undesirably. In another version of the terahertz switch the on/off state is controllable via an external electric bias. ${ }^{55}$ The structure is constructed from gold electric SRRs lithographed on 1- $\mu$ m-thick n-type GaAs substrate. Conductive wires connect the resonators to the biasing source. In the absence of an external voltage, no resonance is observed as the n-type substrate electrically bridges the resonator gaps. Because the gold resonators form a Schottky contact with the substrate, negative biasing depletes free electrons in the substrate around the gap region, leading to the resonance response. Beyond the switch operation, the device is capable of modulating narrow-band terahertz at a kilohertz cycle, limited only by the stray capacitance.

With a high-Q resonance and high electric-field concentration at the surface, metamaterials promise a good sensitivity for biosensing applications. ${ }^{9}$ A sharp resonance is necessary for sensing small changes in the sample dielectric constant, whilst a high field concentration limits the maximum amount of the suspicious sample. An asymmetric double split-ring resonator is designed to achieve both effects at terahertz frequencies. ${ }^{9}$ A numerical study shows that once excited by an electric field the resonator exhibits dual resonances due to coupling between two arcs with different lengths. The resonance shifts considerably after the sample is loaded onto the resonator's surface due to a change in the gap capacitance. Exploiting these resonances for biosensing requires only a fraction of suspected substances in comparison to other resonators. An improved version of the asymmetric double split-ring resonator utilises a rectangular broken ring with sharp tips at the arms. ${ }^{56}$ This rectangular design reduces the overall size of the structure, and the sharp tips results in a higher electric-field confinement. A realisation of this design is shown to be superior to the circular design at the microwave $\mathrm{C}$ band. In another experiment, magnetically activated SRRs operating at terahertz frequencies also indicate a possibility of material sensing through resonance shifting upon dielectric loading. ${ }^{57}$ Recently, a design involving an array of subunit electric SRRs with multiple resonances at terahertz frequencies suggests a possibility of using such a structure for biosensing applications to avoid false-positive detection. ${ }^{58} \mathrm{~A}$ metamaterial which has a set of resonances overlapping with the resonances of a target substance may provide an observable nonlinear behaviour exclusive to that substance. ${ }^{58}$

It would be obvious that terahertz metamaterials have already been developed into some practical terahertz devices, e.g. filters, absorbers, switches, modulators, sensors, with competitive capabilities to existing products. Nonetheless, as discussed earlier, a great deal of challenges at the fundamental level still remain. Overcoming these challenges is critical to enhancement of metamaterial functionalities, and may give way to a new batch of high-efficacy spectacular terahertz structures, including superlenses, hyperlenses, cloaks, etc.

\section{CONCLUSION AND OUTLOOK}

In this paper we have seen how exotic electromagnetic responses can be derived from metamaterials in a desirable way. Metamaterial research is of great importance to terahertz technology, since this new class of material promises strong electromagnetic responses not available from natural materials at terahertz frequencies. Some terahertz devices arising from metamaterial building blocks have been proposed recently. However, improvement of metamaterial performance at this frequency regime has not been fully addressed, and still remains a challenge. Once this basic requisite is satisfied, we can envisage an avalanche of metamaterial applications of terahertz technologies in the areas of astronomy, biochemistry, medicine, and security, for example. 


\section{REFERENCES}

1. J. B. Pendry, "Metamaterials in the sunshine," Nature Materials 5, pp. 599-600, 2006.

2. J. B. Pendry, A. J. Holden, W. J. Stewart, and I. Youngs, "Extremely low frequency plasmons in metallic mesostructures," Physical Review Letters 76(25), pp. 4773-4776, 1996.

3. J. B. Pendry, A. J. Holden, D. J. Robbins, and W. J. Stewart, "Magnetism from conductors and enhanced nonlinear phenomena," IEEE Transactions on Microwave Theory and Techniques 47(11), pp. 2075-2084, 1999.

4. V. Shalaev, "Optical negative-index metamaterials," Nature Photonics 1, pp. 41-48, 2007.

5. V. G. Veselago, "The electrodynamics of substances with simultaneously negative values of $\epsilon$ and $\mu$," Soviet Physics Uspekhi 10(4), pp. 509-514, 1968.

6. J. B. Pendry and D. R. Smith, "Reversing light with negative refraction," Physics Today 57(6), pp. 37-43, 2004.

7. D. R. Smith, W. J. Padilla, D. C. Vier, S. C. Nemat-Nasser, and S. Schultz, "Composite medium with simultaneously negative permeability and permittivity," Physical Review Letters 84(18), pp. 4184-4187, 2000.

8. J. B. Pendry, "Negative refraction makes a perfect lens," Physical Review Letters 85(18), pp. 3966-3969, 2000.

9. C. Debus and P. H. Bolivar, "Frequency selective surfaces for high sensitivity terahertz sensing," Applied Physics Letters 91(18), p. 184102, 2007.

10. M. C. K. Wiltshire, J. B. Pendry, I. R. Young, D. J. Larkman, D. J. Gilderdale, and J. V. Hajnal, "Microstructured magnetic materials for RF flux guides in magnetic resonance imaging," Science 291(5505), pp. 849-851, 2001.

11. J. B. Pendry, D. Schurig, and D. R. Smith, "Controlling electromagnetic fields," Science 312(5781), pp. 1780-1782, 2006.

12. D. Schurig, J. J. Mock, B. J. Justice, S. A. Cummer, J. B. Pendry, A. F. Starr, and D. R. Smith, "Metamaterial electromagnetic cloak at microwave frequencies," Science 314(5801), pp. 977-980, 2006.

13. N. A. Zharova, I. V. Shadrivov, and Y. S. Kivshar, "Inside-out electromagnetic cloaking," Optics Express 16(7), pp. 4615-4620, 2008.

14. J. B. Pendry, A. J. Holden, D. J. Robbins, and W. J. Stewart, "Low frequency plasmons in thin-wire structures," Journal of Physics: Condensed Matter 10, pp. 4785-4809, 1998.

15. M. Bayindir, K. Aydin, E. Ozbay, P. Markoš, and C. M. Soukoulis, "Transmission properties of composite metamaterials in free space," Applied Physics Letters 81(1), pp. 120-122, 2002.

16. I. V. Shadrivov, D. A. Powell, S. K. Morrison, Y. S. Kivshar, and G. N. Milford, "Scattering of electromagnetic waves in metamaterial superlattices," Applied Physics Letters 90(20), art. no. 201919, 2007.

17. T. Weiland, R. Schuhmann, R. B. Greegor, C. G. Parazzoli, A. M. Vetter, D. R. Smith, D. C. Vier, and S. Schultz, "Ab initio numerical simulation of left-handed metamaterials: Comparison of calculations and experiments," Journal of Applied Physics 90(10), pp. 5419-5424, 2001.

18. R. A. Shelby, D. R. Smith, S. C. Nemat-Nasser, and S. Schultz, "Microwave transmission through a twodimensional, isotropic, left-handed metamaterial," Applied Physics Letters 78(4), pp. 489-491, 2001.

19. R. A. Shelby, D. R. Smith, and S. Schultz, "Experimental verication of a negative index of refraction," Science 292, pp. 77-79, 2001.

20. T. A. Klar, A. V. Kildishev, V. P. Drachev, and V. M. Shalaev, "Negative-index metamaterials: Going optical," IEEE Journal of Selected Topics in Quantum Electronics 12(6), pp. 1106-1115, 2006.

21. J. Valentine, S. Zhang, T. Zentgraf, E. Ulin-Avila, D. A. Genov, G. Bartal, and X. Zhang, "Threedimensional optical metamaterial with a negative refractive index," Nature 455, pp. 376-379, 2008.

22. N. Liu, H. Guo, L. Fu, S. Kaiser, H. Schweizer, and H. Giessen, "Three-dimensional photonic metamaterials at optical frequencies," Nature Materials 7, pp. 31-37, 2008.

23. W. Withayachumnankul, G. M. Png, X. X. Yin, S. Atakaramians, I. Jones, H. Lin, B. S. Y. Ung, J. Balakrishnan, B. W.-H. Ng, B. Ferguson, S. P. Mickan, B. M. Fischer, and D. Abbott, "T-ray sensing and imaging," Proceedings of the IEEE 95(8), pp. 1528-1558, 2007. 
24. J. T. Kindt and C. A. Schmuttenmaer, "Far-infrared dielectric properties of polar liquids probed by femtosecond terahertz pulse spectroscopy," Journal of Physical Chemistry 100(24), pp. 10373-10379, 1996.

25. B. M. Fischer, M. Hoffmann, H. Helm, G. Modjesch, and P. U. Jepsen, "Chemical recognition in terahertz time-domain spectroscopy and imaging," Semiconductor Science and Technology 20, pp. S246-S253, 2005.

26. D. M. Mittleman, R. H. Jacobsen, R. Neelamani, R. G. Baraniuk, and M. C. Nuss, "Gas sensing using terahertz time-domain spectroscopy," Applied Physics B: Lasers and Optics 67(3), pp. 379-390, 1998.

27. M. van Exter and D. Grischkowsky, "Optical and electronic properties of doped silicon from 0.1 to 2 THz," Applied Physics Letters 56(17), pp. 1694-1696, 1990.

28. J. Ng and D. Abbott, "Introduction to solid-state quantum computation for engineers," Microelectronics Journal 33(1-2), pp. 171-177, 2002.

29. B. E. Cole, J. B. Williams, B. T. King, M. S. Sherwin, and C. R. Stanley, "Coherent manipulation of semiconductor quantum bits with terahertz radiation," Nature 410, pp. 60-63, 2001.

30. H. S. Brandi, A. Latgé, and L. E. Oliveira, "Rabi oscillations, coherent properties, and model qubits in two-level donor systems under terahertz radiation," Physical Review B 68(23), art. no. 233206, 2003.

31. E. Berry, "Risk perception and safety issues," Journal of Biological Physics 29(2-3), pp. 263-267, 2003.

32. D. M. Mittleman, R. H. Jacobsen, and M. C. Nuss, "T-ray imaging," IEEE Journal of Selected Topics in Quantum Electronics 2(3), pp. 679-692, 1996.

33. Y. C. Shen, T. Lo, P. F. Taday, B. E. Cole, W. R. Tribe, and M. C. Kemp, "Detection and identification of explosives using terahertz pulsed spectroscopic imaging," Applied Physics Letters 86, art. no. 241116, 2005.

34. F. Huang, B. Schulkin, H. Altan, J. F. Federici, D. Gary, R. Barat, D. Zimdars, M. Chen, and D. B. Tanner, "Terahertz study of 1,3,5-trinitro-s-triazine by time-domain and Fourier transform infrared spectroscopy," Applied Physics Letters 85(23), pp. 5535-5537, 2004.

35. B. Ferguson, S. Wang, D. Abbott, and X.-C. Zhang, "Powder retection with THz imaging," in Proc. SPIE Terahertz for Military and Security Applications, 5070, pp. 7-16, 2003.

36. P. R. Coward and R. Appleby, "Development of an illumination chamber for indoor millimeter-wave imaging," in Proc. SPIE Passive Millimeter-Wave Imaging Technology VI and Radar Sensor Technology VII, 5077, pp. 54-61, 2003.

37. C. Zandonella, "T-ray specs," Nature 424, pp. 721-722, 2003.

38. M. van Exter, C. Fattinger, and D. Grischkowsky, "High-brightness terahertz beams characterized with an ultrafast detector," Applied Physics Letters 55(4), pp. 337-339, 1989.

39. D. R. Smith, J. B. Pendry, and M. C. K. Wiltshire, "Metamaterials and negative refractive index," Science 305(5685), pp. 788-792, 2004.

40. T. J. Yen, W. J. Padilla, N. Fang, D. C. Vier, D. R. Smith, J. B. Pendry, D. N. Basov, and X. Zhang, "Terahertz magnetic response from artificial materials," Science 303(5663), pp. 1494-1496, 2004.

41. N. Katsarakis, G. Konstantinidis, A. Kostopoulos, R. S. Penciu, T. F. Gundogdu, M. Kafesaki, E. N. Economou, T. Koschny, and C. M. Soukoulis, "Magnetic response of split-ring resonators in the far-infrared frequency regime," Optics Letters 30(11), pp. 1348-1350, 2005.

42. T. Driscoll, G. O. Andreev, D. N. Basov, S. Palit, T. Ren, J. Mock, S.-Y. Cho, N. M. Jokerst, and D. R. Smith, "Quantitative investigation of a terahertz artificial magnetic resonance using oblique angle spectroscopy," Applied Physics Letters 90(9), art. no. 092508, 2007.

43. W. J. Padilla, M. T. Aronsson, C. Highstrete, M. Lee, A. J. Taylor, and R. D. Averitt, "Electrically resonant terahertz metamaterials: Theoretical and experimental investigations," Physical Review B (Condensed Matter and Materials Physics) 75(4), art. no. 041102, 2007.

44. H.-T. Chen, J. F. O'Hara, A. J. Taylor, R. D. Averitt, C. Highstrete, M. Lee, and W. J. Padilla, "Complementary planar terahertz metamaterials," Optics Express 15(3), pp. 1084-1095, 2007.

45. A. K. Azad, A. J. Taylor, E. Smirnova, and J. F. O'Hara, "Characterization and analysis of terahertz metamaterials based on rectangular split-ring resonators," Applied Physics Letters 92(1), art. no. 011119, 2008.

46. R. Singh, E. Smirnova, A. J. Taylor, J. F. O'Hara, and W. Zhang, "Optically thin terahertz metamaterials," Optics Express 16(9), pp. 6537-6543, 2008. 
47. H. O. Moser, B. D. F. Casse, O. Wilhelmi, and B. T. Saw, "Terahertz response of a microfabricated rodsplit-ring-resonator electromagnetic metamaterial," Physical Review Letters 94(6), art. no. 063901, 2005.

48. B. D. F. Casse, H. O. Moser, L. K. Jian, M. Bahou, O. Wilhelmi, B. T. Saw, and P. D. Gu, "Fabrication of 2D and 3D electromagnetic metamaterials for the terahertz range," Journal of Physics: Conference Series 34, pp. 885-890, 2006.

49. B. D. F. Casse, H. O. Moser, J. W. Lee, M. Bahou, S. Inglis, and L. K. Jian, "Towards three-dimensional and multilayer rod-split-ring metamaterial structures by means of deep x-ray lithography," Applied Physics Letters 90(25), art. no. 254106, 2007.

50. O. Paul, C. Imhof, B. Reinhard, R. Zengerle, and R. Beigang, "Negative index bulk metamaterial at terahertz frequencies," Optics Express 16(9), pp. 6736-6744, 2008.

51. D. Wu, N. Fang, C. Sun, X. Zhang, W. J. Padilla, D. N. Basov, D. R. Smith, and S. Schultz, "Terahertz plasmonic high pass filter," Applied Physics Letters 83(1), pp. 201-203, 2003.

52. H. Tao, N. I. Landy, C. M. Bingham, X. Zhang, R. D. Averitt, and W. J. Padilla, "A metamaterial absorber for the terahertz regime: design, fabrication and characterization," Optics Express 16(10), pp. 7181-7188, 2008.

53. W. J. Padilla, A. J. Taylor, C. Highstrete, M. Lee, and R. D. Averitt, "Dynamical electric and magnetic metamaterial response at terahertz frequencies," Physical Review Letters 96(10), art. no. 107401, 2006.

54. H.-T. Chen, W. J. Padilla, J. M. O. Zide, S. R. Bank, A. C. Gossard, A. J. Taylor, and R. D. Averitt, "Ultrafast optical switching of terahertz metamaterials fabricated on ErAs/GaAs nanoisland superlattices," Optics Letters 32(12), pp. 1620-1622, 2007.

55. H.-T. Chen, W. J. Padilla, J. M. O. Zide, A. C. Gossard, A. J. Taylor, and R. D. Averitt, "Active terahertz metamaterial devices," Nature 444(7119), pp. 597-600, 2006.

56. I. A. I. Al-Naib, C. Jansen, and M. Koch, "Thin-film sensing with planar asymmetric metamaterial resonators," Applied Physics Letters 93(8), art. no. 083507, 2008.

57. T. Driscoll, G. O. Andreev, D. N. Basov, S. Palit, S. Y. Cho, N. M. Jokerst, and D. R. Smith, "Tuned permeability in terahertz split-ring resonators for devices and sensors," Applied Physics Letters 91(6), art. no. 062511, 2007.

58. C. M. Bingham, H. Tao, X. Liu, R. D. Averitt, X. Zhang, and W. J. Padilla, "Planar wallpaper group metamaterials for novel terahertz applications," Optics Express 16(23), pp. 18565-18575, 2008. 